\title{
Zur Bestimmung der Glukuronsäure.
}

\author{
Von \\ Carl Neuberg. \\ (Der Redaktion zugegangen am 16. Mai 1905.)
}

Unter dem gleichlautenden Titel knüpft im letzten Heft dieser Zeitschrift (S. 388) B. Tollens einige Bemerkungen an eine frühere Mitteilung von mir und Neimann ${ }^{1}$ ) über die "quantitative Bestimmung gepaarter Glukuronsäuren». Tollens wendet sich gegen den Passus unserer Arbeit, da $\$$ « die gepaarten Glukuronsäuren bei der Salzsäuredestillation wechselnde und durchaus nicht die theoretischen Mengen Furfurol geben".

Unsere Behauptung stützt sich auf die bisherigen Angaben in der Litteratur. Möglicherweise werden die Furfurolbestimmungen viel genauer durch Anwendung der vervollkommneten Methodik, die Tollens und seine Mitarbeiter inzwischen für die Pentosen ausgearbeitet haben. Früher war gefunden für:

$\begin{array}{lr}\text { Glukuronsäureanhydrid } & 15,23-17,23 \% \text { Furfurol } \\ \text { Euxanthinsäure } & 6,16-7,15 \% \\ \text { Urochloralsäure } & 9,90-10,30 \% \\ \text { Urobutylchloralsaures Kalium } & 8,74-10,27 \%\end{array}$

Dem gegenüber haben wir die von der Theorie für Furfurol * verlangten Werte berechnet:

$\begin{array}{lr}\text { Glukuronsäureanhydrid } & 54,54 \% \\ \text { Euxanthinsäure } & 23,76 \% \\ \text { Urochloralsäure } & 29,48 \% \\ \text { Urobutylchloralsaures Kalium } & 24,52 \%\end{array}$

Diese Gegenüberstellung zeigt, daß die Abweichungen von der Theorie zum Teil erheblich sind, wohl zu bedeutend, um die aus freiem Glukuronsäureanhydrid erhaltene Furfurolausbeute von $17,23 \%$ als Grundlage für die vorgenommene Umrechnung zu wählen.

1) Diese Zeitschrift, Bd. XLIV, S. 127 (1905). 
Aber auch mit dieser Umrechnung bleibt z. B. der für Euxanthinsäure mitgeteilte Wert von 35,7\% Glukuronsäurelakton gegen die Theorie - der verlangte Wert 43,8=100 gesetzt - um ca. 18,5\% zurück, während die Zahl 59,6 für urobutylchloralsaures Kalium 132,7\% der Theorie ergibt.

Wir haben ausdrücklich die Genauigkeit unserer Zuckersäuremethode nur für die physiologisch besonders wichtige Phenolglukuronsäure ${ }^{1}$ ) betont, und damit kommen wir zu dem Kardinalpunkt.

Die Brauchbarkeit der Furfurolmethode für eine angenäherte Bestimmung ${ }^{2}$ ) der in gepaarten Glukuronsäuren enthaltenen Menge Glukuronsäure mag man ohne Einschränkung zugeben, sie kann sich, wie Tollens selbst sagt, immer nur auf die im reinen Zustande abgeschiedenen gepaarten Glukuronsäuren beziehen. Zur quantitativen Bestimmung der letzteren genügt es aber, sie zu wägen, es bedarf dazu keiner Furfuroldestillation. Unsere Versuche sind, wie wir S. 131 ausdrücklich hervorheben, unternommen, um ein für die Harnanalys e brauchbares Verfahren auszuarbeiten oder für physiologische Bedingungen, unter denen gepaarte Glukuronsäuren neben Pentosanen und pentosehaltigen Nucleoproteiden vorkommen. Hier ist, wie Tollens selbst zugibt, seine Methode nicht anwendbar.

Eine auch núr angenähert quantitative Isolierung der gepaarten Glukuronsäuren in Substanz ist in der physiologischen Praxis unmöglich, hier bedarf man gerade eines von dieser Voraussetzung freien Verfahrens. Ein solches haben wir in in dem für die Pathologie wichtigsten Fall, bei der Phenolglukuronsäure, gefunden.

1) Auch bei Mentholglukuronsäure haben wir inzwischen ein befriedigendes Resultat erzielt.

-2) Für vergleichende Zwecke haben wir an anderer Stelle die Brauchbarkeit der bei den Pentosanen hundertfältig erprobten Tollensschen Methode ausdrücklich auch für die Glukuronsäure zugegeben; siehe Neuberg und Neimann, Zeitschrift des Vereins der deutschen Zuckerindustrie, Bd. LV, S. 423. 